\title{
LA ACTIVIDAD HUMANA Y SU IMPACTO SOBRE LOS SISTEMAS DE DRENAJE URBANOS. ESTUDIO DE CASO: LOS DESAGÜES PLUVIALES DE LA CIUDAD DE CORRIENTES.
}

\section{THE HUMAN ACTIVITY AND THEIR IMPACT ON THE URBAN DRAINAGE SYSTEMS. STUDY OF CASE: THE PLUVIAL DRAINAGES OF THE CORRIENTES CITY.}

Ing. Arce, Guillermo Antonio

Docente Investigador CGA -IGUNNE

Prof. Mg. Alberto, Jorge Alfredo

Docente Investigador CGA -IGUNNE

Resumen: La ciudad de Corrientes en las últimas décadas ha presentado un significativo crecimiento poblacional lo que dio lugar a un incremento de las edificaciones y, como consecuencia, un aumento de las superficies impermeables del ejido urbano. Este doble crecimiento trajo aparejado un aumento de las actividades sociales, culturales, comerciales y de servicio, lo cual ha tenido un impacto muy importante sobre el sistema de drenaje pluvial de la ciudad, afectando su funcionamiento y superando en muchos casos las condiciones y solicitaciones para las que fuera diseñado.

Palabras claves: Sistema pluvial, Inundaciones pluviales, Impacto ambiental, vulnerabilidad socio ambiental.

\begin{abstract}
Corrientes city in the last decades has presented a significant population growth what gave place to an increment in the constructions, as consequence, an increase of the waterproof surfaces of the urban public land. This double growth brought harnessed an increase of the social, cultural, commercial activities and of service, that which has had a very important impact on the system of pluvial drainage of the city, affecting its operation and overcoming in many cases the conditions and solicitations for those that it was designed.
\end{abstract}

Keywords: pluvial system, pluvial floods, environmental impact, vulnerability partner.

\section{Introducción}

En las últimas décadas la ciudad de Corrientes ha presentado un importante crecimiento poblacional que dio lugar a un marcado incremento en las edificaciones, lo cual ha tenido un impacto muy importante sobre el sistema de drenaje pluvial de la ciudad, afectando su funcionamiento y superando en muchos casos la capacidad de repuesta para la que fuera diseñado.

Una de las consecuencias de la convergencia de factores arriba mencionados son las inundaciones provocadas por las lluvias de cierta intensidad (por ejemplo $60 \mathrm{~mm} / \mathrm{hora}$ ) y agravadas debido a la drástica reducción de la capacidad de almacenamiento de los reservorios naturales (lagunas y superficies de absorción), por relleno de las mismas y/o por ocupación de sus márgenes; a los que se suman las inundaciones originadas por una insuficiente capacidad de los conductos de drenaje pluviales.

Si bien ambas problemáticas tienen una duración de pocas horas, producen graves trastornos al normal funcionamiento de la ciudad. Estas inundaciones son difíciles de resolver sólo con medidas estructurales (obras), por los condicionantes geomorfológicos (topografía, suelos e hidrografía) propios del emplazamiento de la ciudad, así como, por la dificultad de conseguir financiamiento para realizarlas.

Uno de los mayores problemas, posibles de superar, en los sistemas de conducción pluviales urbanos es el acarreo de basura y sedimentos en las bocas de tormenta y conductos, que obstruyen el escurrimiento provocando inundaciones.

Publicado en formato digital: Ing. Guillermo Antonio Arce y Prof. Mgter. Jorge Alfredo Alberto. LA ACTIVIDAD HUMANA Y SU IMPACTO SOBRE LOS SISTEMAS DE DRENAJE URBANOS. ESTUDIO DE CASO: LOS DESAGÜES PLUVIALES DE LA CIUDAD DE CORRIENTES. Revista Geográfica Digital. IGUNNE. Facultad de Humanidades. UNNE. Año 8. № 16. Julio - Diciembre 2011. ISSN 16685180 Resistencia, Chaco. En: http://hum.unne.edu.ar/revistas/geoweb/default.htm 


\section{Características Generales}

La ciudad de Corrientes está ubicada sobre la margen izquierda del Río Paraná cercana a la confluencia con el Río Paraguay. El Departamento capital abarca una superficie de $522 \mathrm{~km}^{2}$ y limita al Norte y al Oeste con el Río Paraná, al Este con los Departamentos de San Cosme y San Luis y al Sur con el Departamento de Empedrado.

Comprende unas 3.000 manzanas incluyendo zonas urbanas y suburbanas. Tiene una participación porcentual en el total de la población donde el Departamento Capital ha seguido un proceso de concentración a expensas de la población provincial con un mayor incremento que representa un $22,7 \%$ en el periodo $1991-2001$.

Si se considera el aglomerado urbano que comprende el Gran Corrientes, para mitad del 2009 su población se estimaba aproximadamente en 354.000 habitantes (INDEC, 2009), datos que la convierten en el $2^{\circ}$ aglomerado urbano del NEA y el $13^{\circ}$ a nivel nacional.

Desde el punto de vista geomorfológico la ciudad de Corrientes se encuentra inserta dentro de la denominada por el Dr. Popolizio $(1977,1997)$ "Gran Región Occidental de la Provincia de Corrientes", es una gran planicie sedimentaria, comprendida entre el Río Paraná y la depresión del Iberá. Las pendientes son muy bajas, el escurrimiento es lento. Está conformado por una serie de lomadas y depresiones en las que se insertan ríos que desaguan en el Paraná. Los suelos son oscuros y muy orgánicos en las depresiones y arenosos rojizos o amarillentos con incrustaciones calcáreas en las terrazas de los mismos.

Frente a la ciudad de Corrientes el ancho del río Paraná es de unos $4.000 \mathrm{~m}$ y la planicie de inundación tiene unos $13 \mathrm{~km}$. El régimen del río es muy regular y presenta un marcado incremento de caudal durante los meses de verano y estiajes durante los meses invierno. Los estudios realizados indican que el Paraná no presenta fenómenos de contaminación que puedan afectar en forma notable la calidad de sus aguas; esto se debe a la capacidad de dilución y autodepuración que presenta este curso.

En lo que hace a los cuerpos de agua que cruzan el ejido urbano, cabe mencionar los siguientes: arroyo Salamanca, Poncho Verde, Limita, Santo Domingo y Laguna Seca. Todos ellos se hallan muy antropizados conformando actualmente parte del sistema de drenaje de la ciudad por entubamiento o escurrimiento a cielo abierto o canales.

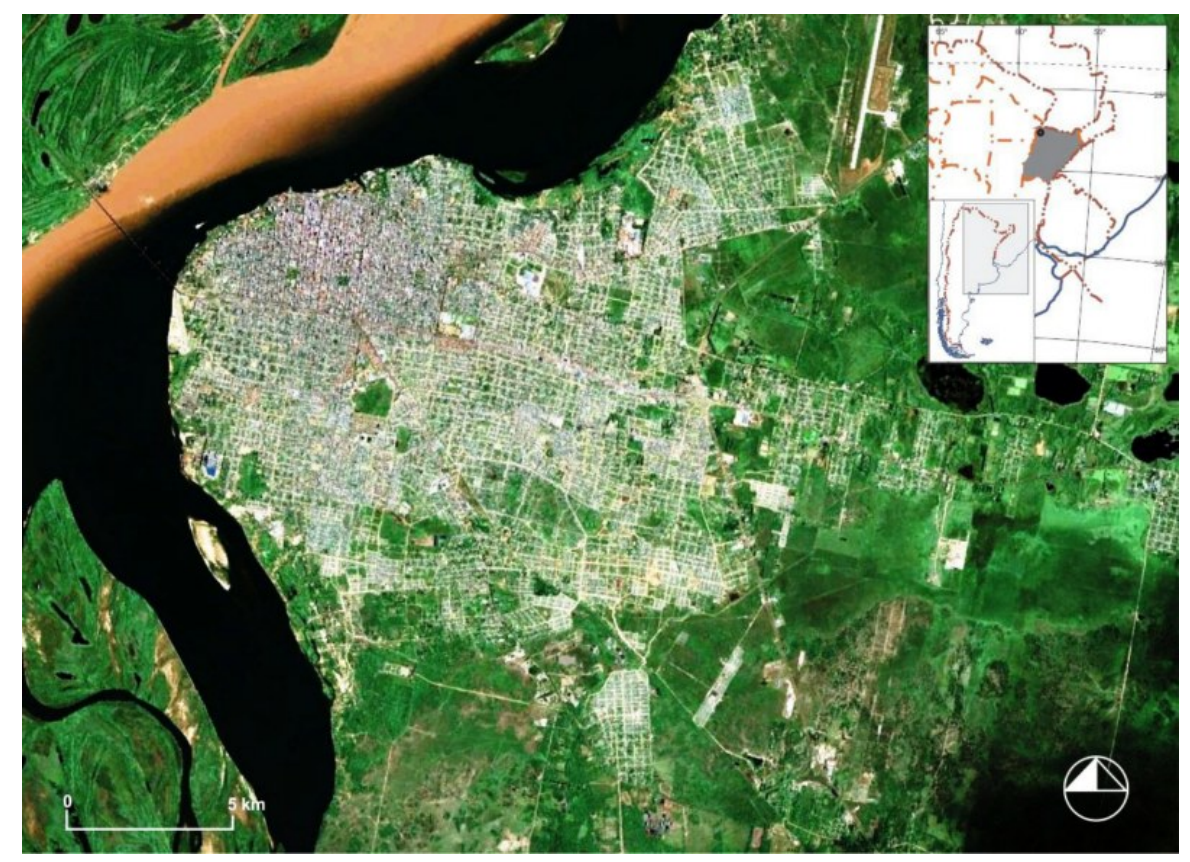

Fig. 1. Corrientes Capital se encuentra sobre la margen izquierda del Río Paraná que junto al Gran Resistencia que se localiza en la margen opuesta, pero unidas por el puente Gral Belgrano, conforman una conurbación de gran relevancia socio-económica para la región NEA. (Fuente: Google Ehart, fecha de consulta 3 de noviembre 2011).

Publicado en formato digital: Ing. Guillermo Antonio Arce y Prof. Mgter. Jorge Alfredo Alberto. LA ACTIVIDAD HUMANA Y SU IMPACTO SOBRE LOS SISTEMAS DE DRENAJE URBANOS. ESTUDIO DE CASO: LOS DESAGÜES PLUVIALES DE LA CIUDAD DE CORRIENTES. Revista Geográfica Digital. IGUNNE. Facultad de Humanidades. UNNE. Año 8. № 16. Julio - Diciembre 2011. ISSN 16685180 Resistencia, Chaco. En: http://hum.unne.edu.ar/revistas/geoweb/default.htm 


\section{Condicionantes de la degradación del sistema de desagüe pluvial urbano}

Entre las posibles causas que desencadenan la degradación del sistema de desagüe pluvial urbano pueden contarse las siguientes actividades humanas como indicadores de presión:

1. Basura arrojada a la vía pública al transitar (Fig. 2)

2. Basura depositada fuera de lugar u horario de recolección (Basurales espontáneos o crónicos - actividades de cartoneros, carritos y otros agentes de reciclado informales) (Fig. 2)

3. Tareas de barrido y limpieza con acumulación de basura en zonas próximas a los sumideros o bocas de tormenta (Fig. 3)

4. Obras en construcción de índole pública (impermeabilización de calles y paseos - Ejecución de parterres sobre elevados que propician erosión de suelos).

5. Obras de empresas de servicios (agua, cloaca, telefonía, fibra óptica, etc., que realizan excavaciones y depositan material sobre calles y veredas) (Fig. 4)

6. Obras en construcción privadas (depósito de materiales para construcción en veredas y calles - preparación de mezclas cementicias sobre la calzada - impermeabilización creciente de veredas y patios internos - aumento de la construcción de edificaciones en altura que aumentan la superficie impermeable del ejido urbano).

7. Tareas de mantenimiento de pluvial inadecuadas.

8. Actividades comerciales y de servicio (producción de enorme cantidad de residuos sólidos y líquidos que van desde papeles, cajas de cartón, utensilios descartables, envases no retornables, incluyendo residuos orgánicos sólidos, grasas y líquidos como combustibles, aceites, barros saturados, detergentes, solventes, etc.).

9. Actividades de publicidad, propaganda y marketing (producción de revistas, catálogos, folletos, pegatina de carteles que generan basura en la vía pública).

10. Actividades culturales al aire libre, eventos deportivos y musicales (que generan aglomeración y producción de basura en la vía pública asociadas a actividades comerciales de gastronomía, publicidad y marketing).

11. Actividades políticas y proselitistas (producción de basura en actos masivos - pegatina de carteles, reparto de folletería y boletas para votación).

12. Conexiones clandestinas de cloaca y otros sin tratamiento

13. Erosión de calles de tierra y parterres de paseos públicos (ácidos, solventes, pinturas, etc.)

14. Drenaje de cunetas y zanjas de calles de tierra (que aportan residuos inorgánicos y orgánicos por falta de conexión al sistema cloacal)

15. Productos de la actividad biológica de animales y plantas (hojas, flores, ramas, etc.) (Fig. 5)

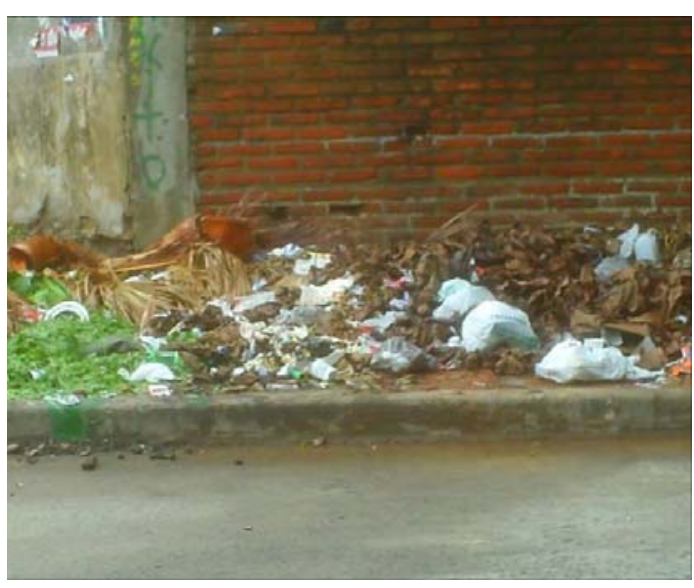

Fig. 2. Drenaje deficiente por desarrollo de micro basurales en la vía pública.

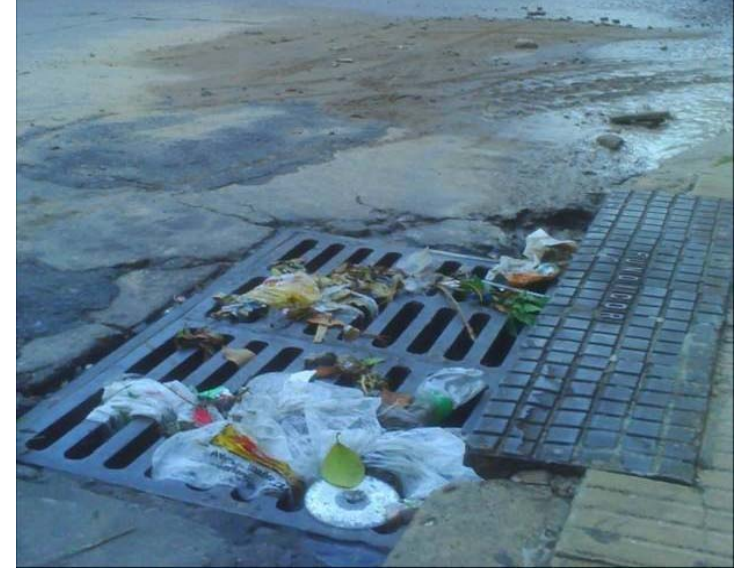

Fig. 3. Drenaje deficiente por acumulación de basura en sumideros o boca de tormenta.

Publicado en formato digital: Ing. Guillermo Antonio Arce y Prof. Mgter. Jorge Alfredo Alberto. LA ACTIVIDAD HUMANA Y SU IMPACTO SOBRE LOS SISTEMAS DE DRENAJE URBANOS. ESTUDIO DE CASO: LOS DESAGÜES PLUVIALES DE LA CIUDAD DE CORRIENTES. Revista Geográfica Digital. IGUNNE. Facultad de Humanidades. UNNE. Año 8. № 16. Julio - Diciembre 2011. ISSN 16685180 Resistencia, Chaco. En: http://hum.unne.edu.ar/revistas/geoweb/default.htm 


\begin{tabular}{|l|l|}
\hline Revista Geográfica Digital. IGUNNE. Facultad de Humanidades. UNNE. Año 8. № 16. Julio- Diciembre \\
2011. ISSN 1668-5180 Resistencia, Chaco
\end{tabular}

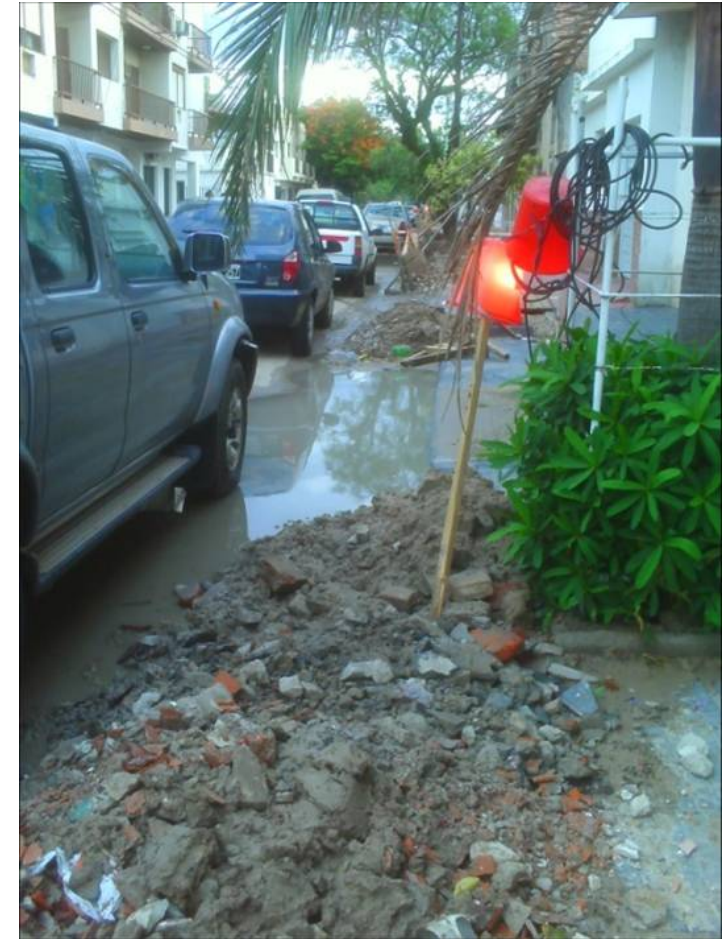

Fig. 4. Drenaje deficiente por excavaciones y depósito de materiales sobre calles y veredas.

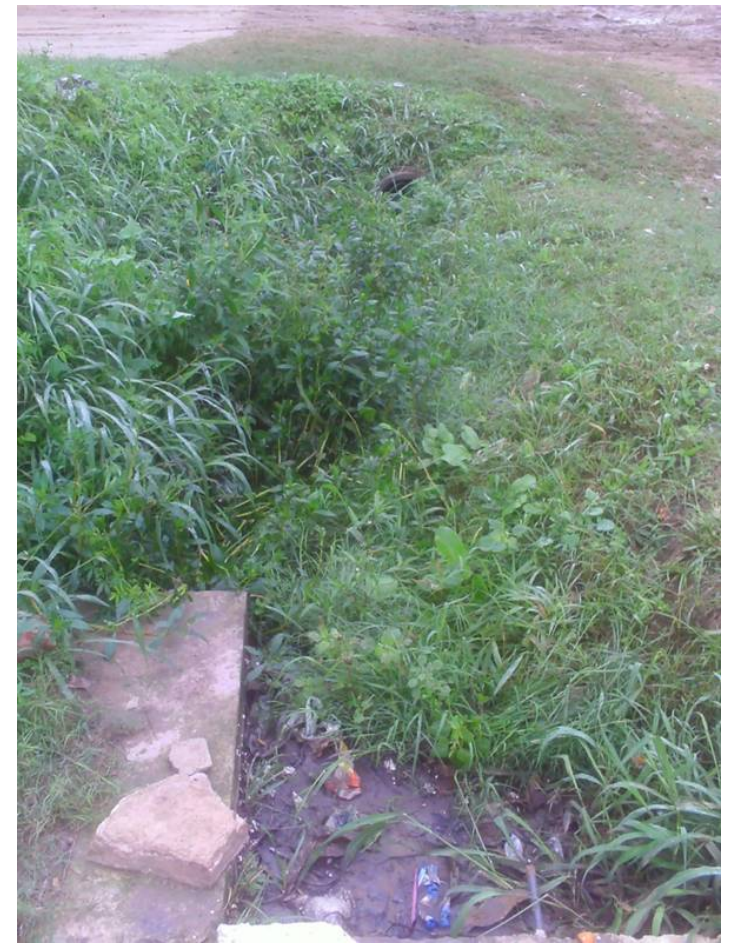

Fig. 5. Drenaje deficiente por actividad biológica acentuada por conexiones clandestinas domiciliarias a zanjas o cunetas.

\section{Problemáticas derivadas de la degradación del sistema de desagüe pluvial}

Entre las posibles consecuencias derivadas de los condicionantes expuestos con anterioridad pueden detallarse las siguientes problemáticas:

1. Permanencia del agua en las calles aún después de la precipitación pluvial por obstrucción de sumideros y conductos.

2. Inundaciones urbanas con montos pluviométricos menores a los valores correspondientes al diseño del desagüe pluvial (Fig. 6).

3. Insalubridad y aumento de riesgo de enfermedades asociadas con la basura acumulada en cámaras y conductos (Fig. 7).

4. Deterioro precoz de los conductos pluviales por ampliaciones fuera de las de diseño.

5. Falla de conductos por pérdida de capacidad portante por erosión del hormigón y/o corrosión de la malla de acero estructural (Fig. 8).

6. Aumento en los costos de servicios de mantenimiento de pluviales por obstrucciones con basura (Fig. 9).

7. Reducción de las secciones útiles de los desagües y presencia de obstrucciones parciales y/o totales (Fig. 10).

8. Desagradado estético y visual por acumulación de basura en las bocas de tormenta (Fig. 11).

9. Malos olores y contaminación atmosférica con gases (metano entre otros) de descomposición orgánica (Fig. 11)

10. Aumento de insectos y alimañas asociadas a la basura y malos olores que manan de los desagües.

Publicado en formato digital: Ing. Guillermo Antonio Arce y Prof. Mgter. Jorge Alfredo Alberto. LA ACTIVIDAD HUMANA Y SU IMPACTO SOBRE LOS SISTEMAS DE DRENAJE URBANOS. ESTUDIO DE CASO: LOS DESAGÜES PLUVIALES DE LA CIUDAD DE CORRIENTES. Revista Geográfica Digital. IGUNNE. Facultad de Humanidades. UNNE. Año 8. № 16. Julio - Diciembre 2011. ISSN 16685180 Resistencia, Chaco. En: http://hum.unne.edu.ar/revistas/geoweb/default.htm 


\begin{tabular}{|l|l|}
\hline Revista Geográfica Digital. IGUNNE. Facultad de Humanidades. UNNE. Año 8. № 16. Julio- Diciembre \\
2011. ISSN 1668-5180 Resistencia, Chaco
\end{tabular}

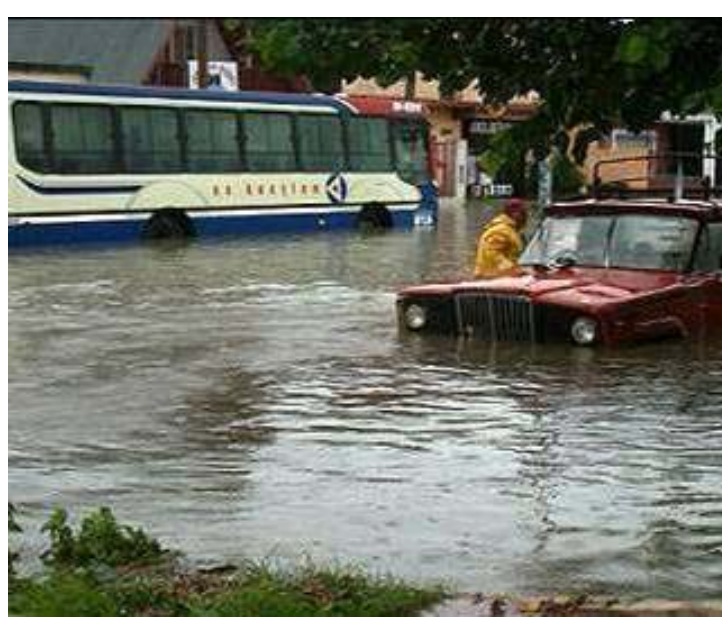

Fig. 6. Inundaciones urbanas por precipitaciones en un corto periodo de tiempo



Fig. 8. Deterioro de los conductos pluviales

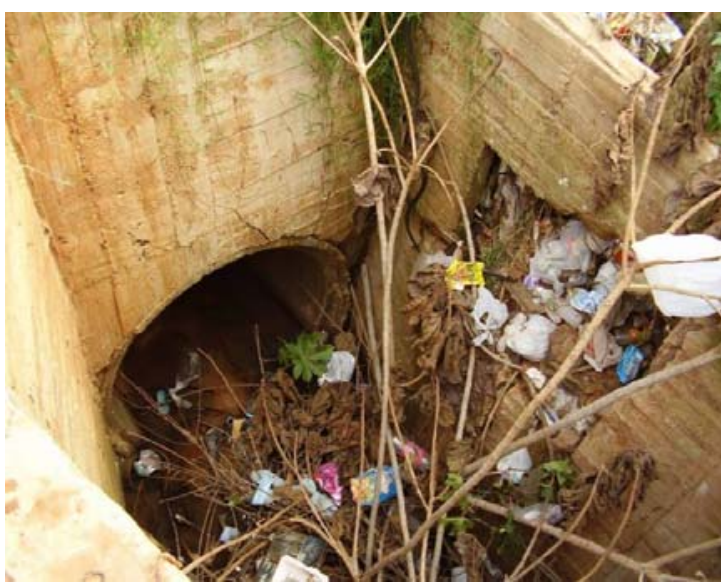

Fig. 10. Presencia de obstrucciones parciales y/o totales de los conductos pluviales



Fig. 7. Insalubridad y aumento de riesgo de enfermedades

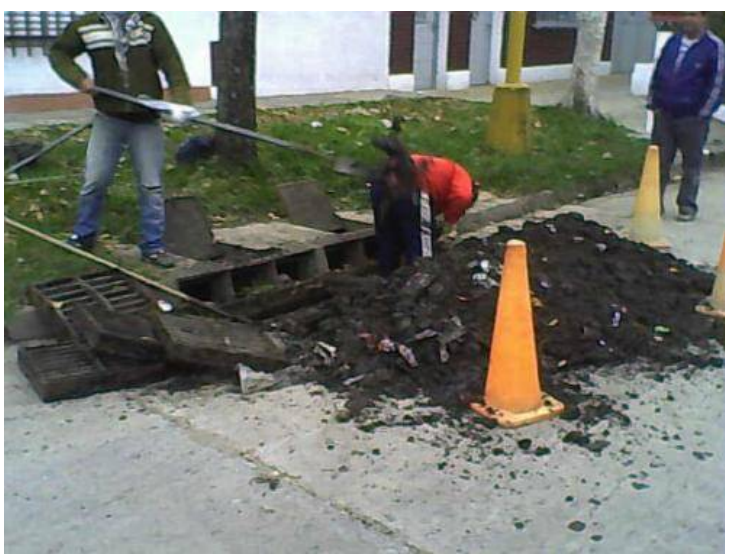

Fig. 9. Aumento en los costos de servicios de mantenimiento.

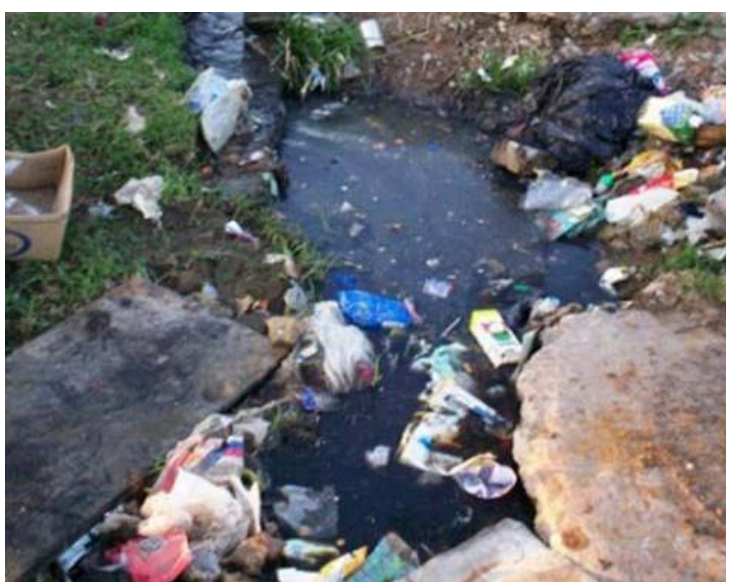

Fig. 11. Desagradado estético y visual a lo que se suman malos olores por descomposición orgánica

Publicado en formato digital: Ing. Guillermo Antonio Arce y Prof. Mgter. Jorge Alfredo Alberto. LA ACTIVIDAD HUMANA Y SU IMPACTO SOBRE LOS SISTEMAS DE DRENAJE URBANOS. ESTUDIO DE CASO: LOS DESAGÜES PLUVIALES DE LA CIUDAD DE CORRIENTES. Revista Geográfica Digital. IGUNNE. Facultad de Humanidades. UNNE. Año 8. № 16. Julio - Diciembre 2011. ISSN 16685180 Resistencia, Chaco. En: http://hum.unne.edu.ar/revistas/geoweb/default.htm 


\section{Medidas de mitigación a la problemática expuesta}

La mitigación se define como cualquier medida sostenida que se adopte para reducir o eliminar riesgos a largo plazo para la vida humana y los bienes materiales provenientes de sucesos de una problemática socio ambiental de gran envergadura como lo es la degradación de los sistemas de desagües pluviales.

La mitigación, estimula la reducción a largo plazo de la vulnerabilidad a los riesgos. La meta de la mitigación es disminuir la necesidad de respuesta, a diferencia de simplemente aumentar la capacidad de respuesta.

La mitigación puede salvar vidas y reducir los daños materiales, además de ser rentable y ambientalmente sensata. Esto, a su vez, reduce el enorme costo de los desastres para los propietarios de inmuebles y todos los niveles de gobierno.

Además, la mitigación protege instalaciones críticas de las comunidades, reduce el riesgo de quedar sujeto a responsabilidad legal y disminuye los trastornos a las comunidades. Para ello se debe tener en cuenta el siguiente marco legal como indicador de respuesta a la situación problema presentada en este trabajo:

- Ordenanza Municipal 1071 Código de Planeamiento Urbano y Modificatorias

- Ordenanza Municipal 1623 Código de Edificación

- Ordenanza Municipal 2400 Modificación Ord. 1623 Código de Edificación

- Ordenanza Municipal 2466 Reglamento Uso de Suelo

- Ordenanza Municipal 4382 Habilitación Comercial y Anexos

- Ordenanza Municipal 2402 Instalación de Lavaderos de Vehiculos

- Ordenanza Municipal 2132 Reglamento sobre Pensiones (Vivienda Comunitaria)

- Ordenanza Municipal 3686 Aprobación de Planos de Obra

- Ordenanza Municipal 3581 Inspección de Obra Pública

- Ley 25612/02 Presupuestos mínimos de protección ambiental sobre la gestión integral de residuos de origen industrial y de actividades de servicio

- Ley 25688/02 Presupuestos mínimos ambientales para la preservación de las aguas, su aprovechamiento y uso racional. Se crean los comités de cuencas hídricas.

- Ley 25916/04 Presupuestos mínimos de protección ambiental para la gestión de residuos domiciliarios.

- Decreto 674/89 Reemplaza al Decreto 2125/78 Principio Contaminador-Pagador (pago de cuota de resarcimiento por parte de aquellos que vuelcan efluentes industriales a las aguas lindantes).

- Ley 25.675 - Ley de política ambiental

- Ley 25.688- Régimen de gestión de aguas

- Ley 24.051 - Residuos peligrosos

- Ley 25.612- Gestión de residuos industriales y de servicios

- Ley 25.670 - Gestión de PCB's

- Ley 25.916 - Gestión de residuos domiciliarios

Ante la pregunta ¿Qué se puede hacer? Se proponen algunas ideas o principios rectores para gestionar las inundaciones urbanas a partir de medidas de control y/o mitigación que pueden dividirse en Estructurales (obras civiles) y no Estructurales.

\section{Medidas estructurales y no estructurales de control ylo mitigación}

Las denominadas medidas estructurales y no estructurales de control y/o mitigación tienen un claro sentido de atenuación del impacto final del escurrimiento superficial, apartándose del criterio tradicional de arbitrar soluciones por la única vía de incrementar las dimensiones de las estructuras de evacuación. Buscan trasladar espacial y temporalmente parte de la inevitable conversión de lluvia incidente en escorrentía superficial que se opera en las cuencas. Así, se intenta incrementar las componentes de retención superficial, o las tasas de infiltración, con el consecuente aumento de las de escurrimiento subsuperficial o percolación profunda.

Comprenden un conjunto de medidas de aplicación a un nivel distribuido, es decir, en todo el ámbito de la cuenca, sean estos en parcelas dentro de las áreas de manzanas, o en espacios

Publicado en formato digital: Ing. Guillermo Antonio Arce y Prof. Mgter. Jorge Alfredo Alberto. LA ACTIVIDAD HUMANA Y SU IMPACTO SOBRE LOS SISTEMAS DE DRENAJE URBANOS. ESTUDIO DE CASO: LOS DESAGÜES PLUVIALES DE LA CIUDAD DE CORRIENTES. Revista Geográfica Digital. IGUNNE. Facultad de Humanidades. UNNE. Año 8. № 16. Julio - Diciembre 2011. ISSN 16685180 Resistencia, Chaco. En: http://hum.unne.edu.ar/revistas/geoweb/default.htm 
de la vía pública (aceras y calzadas). Su instrumentación es viable a través de regulaciones que se establecen mediante normas de planeamiento urbano, y de la construcción en general sobre espacios de dominio público y privado.

\section{A. Medidas estructurales de control}

- Construcción de canteros y arborización: La implementación de canteros en todas las veredas con un ancho promedio de $1 \mathrm{~m}$, representaría $4 \%$ de área permeable. Para cumplir con la función de captadores y retardadores de escurrimiento, no deberían tener cordones perimetrales y sus respectivos niveles deberían ser inferiores a los de las veredas. Los ya existentes deberían adecuarse eliminando, los cordones perimetrales. La eficiencia hidrológica de los canteros podría ser potenciada implementando un programa de arborización de los mismos. Con ello se lograría disminuir el coeficiente de impermeabilización, minimizar erosión, mejorar absorción y retardar escurrimiento superficial (Fig. 12).

- Modificación de espacios verdes y parterres: Con puntuales excepciones, los espacios verdes, parterres y canteros de la ciudad, desaguan superficialmente hacia las calles aumentando el caudal de escurrimiento y agravando la problemática de inundación. La modificación de las pendientes para que actúen como retardadores y captores de escurrimiento contribuiría a, evitar erosión, disminuyendo el aporte de residuos orgánicos e inorgánicos a las calles (Fig. 13 y Fig. 15).

- Construcción de retardadores de escurrimiento en los lotes: con lo cual se intenta controlar los excedentes hídricos en la fuente, o sea en los propios lotes (Fig. 14).

- Mantenimiento de sumideros libres de obstrucciones: Esta tarea deberá ser sistemática y planificada abandonando la actuación usual, de medida estacional o de emergencia.

- Ejecución de obras pluviales: Implicaría la gestión de fondos para la ejecución del Plan Director de pluviales de la ciudad y la reparación de la estructura existente.

\section{B. Medidas no estructurales (de gestión y control)}

- Creación de un Área responsable del sistema de drenaje: A nivel municipal no existe en la actualidad un área específica que atienda esta cuestión y las responsabilidades quedan diluidas entre varias divisiones, lo cual resulta en la falta de atención programática y continua y en la falta de planificación y gestión de medidas y controles.

- Planificación del mantenimiento del sistema de drenaje: Normalmente esta tarea está ausente en la Municipalidad de Corrientes. Y todas las actuaciones se centran en dar respuesta a situaciones problemáticas "a posteriori" de la ocurrencia de fenómenos de inundación, de manera no planificada, atendiendo problemas puntuales sin considerar la totalidad del sistema y dando respuestas precarias y provisorias para salvar la emergencia.

- Actualización del Plan Director de desagües pluviales: Si bien la ciudad cuenta con un Plan Director, el mismo es de la década del 80. El mismo no contempla situaciones actuales existentes, tales como nuevos barrios, invasión de humedales y lagunas, prolongación de la avenida costanera, desmesurado crecimiento de edificación en altura en el casco céntrico, etc. Además centra el programa de gestión en medidas estructurales, y no contempla la aplicación de medidas no estructurales, sin mencionar la escasa atención a los problemas medio ambientales.

- Actualización de la normativa: Para introducir reglamentaciones tendientes a crear medidas de mitigación de impacto hídrico en lotes particulares (canteros en veredas, trincheras de piedra en patios internos, conservación de áreas permeables dentro de las parcelas). A nivel del uso del espacio público ( $12 \%$ de la superficie total) deberían incorporarse normativas tendientes a que las mismas actúen como reservorios o retardadores.

- Creación de normativa: nueva ordenanza municipal, atendiendo el concepto de Impacto Hídrico Cero (similar ordenanza № 5403 - Ciudad Resistencia - Chaco) y creando incentivos impositivos para los vecinos que ejecuten medidas estructurales de mitigación en sus lotes.

Publicado en formato digital: Ing. Guillermo Antonio Arce y Prof. Mgter. Jorge Alfredo Alberto. LA ACTIVIDAD HUMANA Y SU IMPACTO SOBRE LOS SISTEMAS DE DRENAJE URBANOS. ESTUDIO DE CASO: LOS DESAGÜES PLUVIALES DE LA CIUDAD DE CORRIENTES. Revista Geográfica Digital. IGUNNE. Facultad de Humanidades. UNNE. Año 8. № 16. Julio - Diciembre 2011. ISSN 16685180 Resistencia, Chaco. En: http://hum.unne.edu.ar/revistas/geoweb/default.htm 


\begin{tabular}{|l|l|}
\hline 2011. ISSN 1668-5180 Resistencia, Chaco \\
2015ta Geográfica Digital. IGUNNE. Facultad de Humanidades. UNNE. Año 8. № 16. Julio- Diciembre
\end{tabular}

\section{No estructurales (de educación y participación)}

- Programa de promoción y propaganda: que den a conocer al público las tareas de control y administración planteadas además de dar una fuerte promoción a los aspectos negativos de la basura sobre el sistema de drenaje y su relación con las inundaciones urbanas

- Programa de educación: (carteles - avisos en medios - páginas de internet - visita de escuelas a zonas en que las medidas de control fueron implementadas). Resaltar la conexión entre los problemas de inundación y la basura arrojada a la vía pública y otras actividades contaminantes.

- Programa de capacitación: Normalmente, la capacitación está dirigida al equipo político de la gestión, los cuales sólo participan de la toma de decisiones durante períodos cortos haciendo poco efectiva su actuación, con el agravante de la no transmisión de conocimientos a agentes de la planta permanente. Los agentes y funcionarios de carrera de la municipalidad deberán ser capacitados en la filosofía de enfoque sistémico de la gestión de aguas de lluvia. Esta capacitación debe incluir necesariamente, al cuadro de inspectores de tránsito y guardia ciudadana que no atienden cuestiones preventivas referidas a arrojar basura a la vía pública por parte de peatones y conductores de vehículos.

- Programa de participación: Invitar a vecinos a colaborar con la mitigación del impacto hídrico en sus propios lotes a través de regímenes de promoción impositiva.

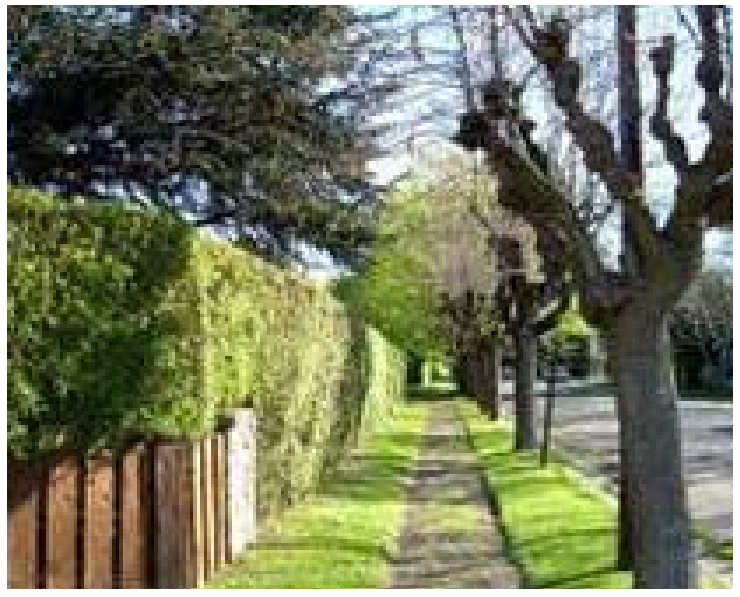

Fig. 12. Construcción de canteros y arborización.

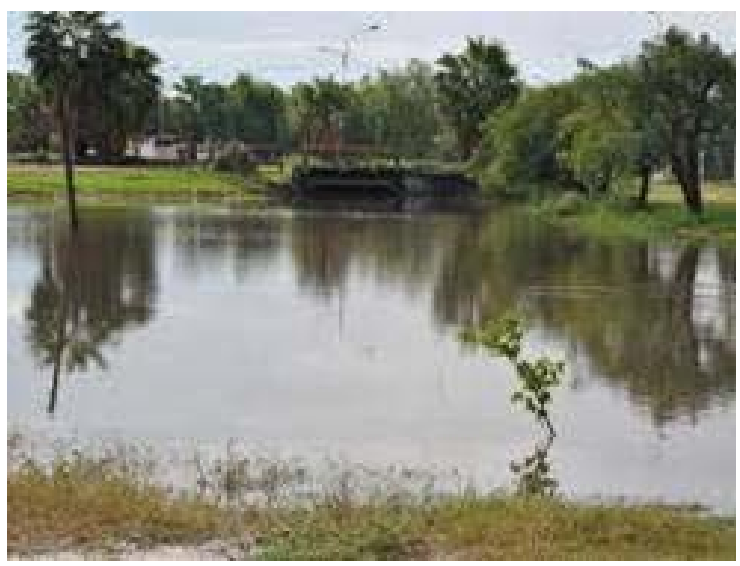

Fig. 14. Construcción de retardadores de escurrimiento en los lotes.

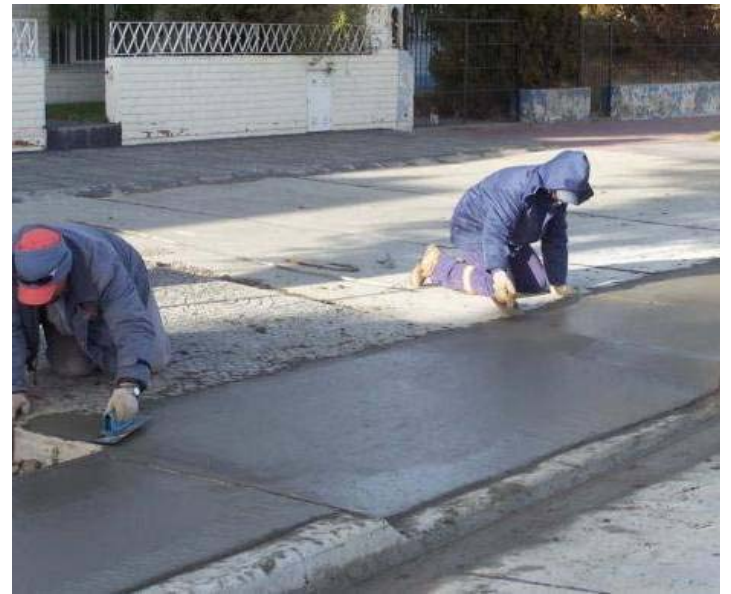

Fig. 13. Limitar la modificación de espacios verdes y parterres como se muestra en la fotografía.

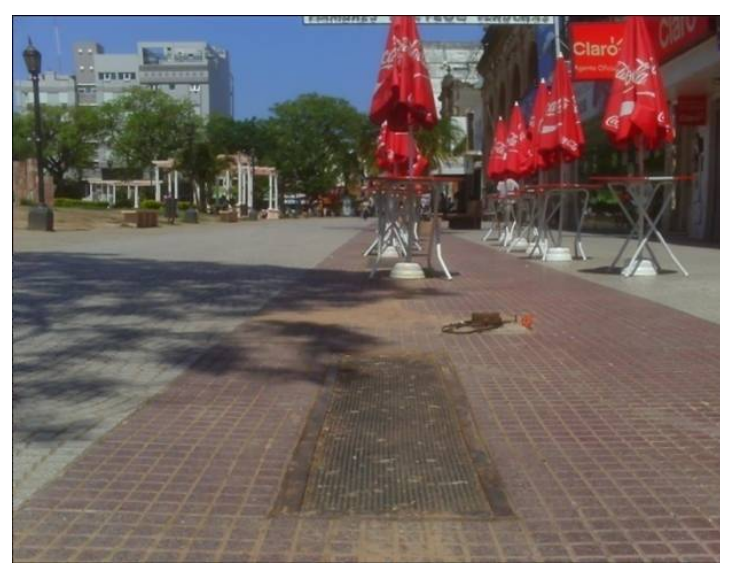

Fig. 15. Limitar el desarrollo de superficies impermeables semejante al de la fotografía.

Publicado en formato digital: Ing. Guillermo Antonio Arce y Prof. Mgter. Jorge Alfredo Alberto. LA ACTIVIDAD HUMANA Y SU IMPACTO SOBRE LOS SISTEMAS DE DRENAJE URBANOS. ESTUDIO DE CASO: LOS DESAGÜES PLUVIALES DE LA CIUDAD DE CORRIENTES. Revista Geográfica Digital. IGUNNE. Facultad de Humanidades. UNNE. Año 8. № 16. Julio - Diciembre 2011. ISSN 16685180 Resistencia, Chaco. En: http://hum.unne.edu.ar/revistas/geoweb/default.htm 


\section{A modo de conclusión}

Las medidas de mitigación son un conjunto de acciones para aminorar o eliminar el impacto de las amenazas naturales acentuadas por la acción antrópica, mediante la reducción de la vulnerabilidad física, funcional o social del sistema. El desarrollo del proceso de urbanización que se observó en los últimos años anticipa un escenario complejo en el crecimiento en la problemáticas tratadas sobre el sistema de desagües pluviales. Esto conduciría a la necesidad de profundizar normativas de control a partir de las siguientes recomendaciones:

- Existencia de marco legal: A nivel nacional y provincial, existen leyes y normas con un distinto nivel de aplicación, que pueden ser ajustadas perfectamente a la problemática de actividades contaminantes de la vía pública y su consecuencia sobre el sistema de drenaje.

- Insuficiencia de la normativa: Las ordenanzas municipales respecto del mantenimiento de la limpieza de la ciudad regula sólo la actuación del municipio. Con referencia a la responsabilidad de los privados sobre el cuidado de la limpieza sólo afecta a los poseedores u ocupantes de lotes según el uso de suelo y actividad que se desarrolle en los mismos. No se hace referencia a los agentes privados, productores de basura en espacios públicos ni se contemplan sanciones o medidas preventivas para esta situación. Tampoco existen ordenanzas específicas sobre tareas de mitigación y prevención de inundaciones.

- Procedimiento administrativo: Uno de los mayores problemas que enfrenta la municipalidad de Corrientes es la ausencia de un área específica dentro del organigrama de funciones, que atienda esta cuestión. La responsabilidad recae por separado entre las Direcciones de Planificación, Medio Ambiente, Defensa Civil y Obras Públicas. Todas ellas con injerencia en alguna actividad de planificación, mantenimiento y ejecución pero sin determinación específica los alcances de su actuación en tareas preventivas y de emergencia. Tampoco está claro el nivel de coordinación de estas áreas, en las distintas etapas de un sistema de gestión de la red de drenaje. La situación anterior conlleva a actuaciones sobre la emergencia, de manera no planificada, dando respuestas puntuales de manera precaria y provisoria con el sólo objeto de atender la urgencia sin considerar la totalidad del sistema de drenaje, ni soluciones sostenibles a mediano y largo plazo.

Si bien existe una ley provincial que establece como organismo de aplicación al ICAA (Instituto Correntino del Agua y del Ambiente), hasta la fecha no se han realizado verificaciones de impacto ambiental u otros controles, de las obras de pluvial nuevas ejecutadas por el propio municipio en ninguna de sus etapas (desde proyecto a ejecución). La tarea de verificación y contralor del mantenimiento y estado de la infraestructura tampoco tiene un área de responsabilidad específica por lo que no se realizan tareas de mantenimiento en forma programática.

\section{Bibliografía}

- Foschiatti, A. Maria (Eds.) 2008. Aportes conceptuales y empíricos de la vulnerabilidd global. Resistencia. EUDENE. UNNE. Resistencia. Argentina. pp. 425.

- Franchini, T. y Rey, W. 1986. Las inundaciones en el Nordeste Argentino. Boletín de Medio Ambiente y Urbanización. Año 5, n 19. Clacso. Bs As.

- INDEC. 2009. Encuesta permanente de hogares. Estimación población aglomerados urbanos de Argentina para 2009. (en español) (PDF) págs. 3. http://www.indec.gov.ar/nuevaweb/cuadros/4/EPHcont_3trim09.pdf. Consultado el 2610-2011.

- Bianucci, S. P. et Al. 2004. Gestión para la emergencia en situaciones de anegamiento urbano. Comunicaciones Científicas y Tecnológicas 2004. SGCyT/UNNE. http://www.unne.edu.ar/Web/cyt/com2004/7-Tecnologia/T-028.pdf Consultado el 31-10-2011

Publicado en formato digital: Ing. Guillermo Antonio Arce y Prof. Mgter. Jorge Alfredo Alberto. LA ACTIVIDAD HUMANA Y SU IMPACTO SOBRE LOS SISTEMAS DE DRENAJE URBANOS. ESTUDIO DE CASO: LOS DESAGÜES PLUVIALES DE LA CIUDAD DE CORRIENTES. Revista Geográfica Digital. IGUNNE. Facultad de Humanidades. UNNE. Año 8. № 16. Julio - Diciembre 2011. ISSN 16685180 Resistencia, Chaco. En: http://hum.unne.edu.ar/revistas/geoweb/default.htm 
- Campana, N., Mendiondo, M. y Tucci, C. 1995: Una aproximación multipropósito para la estimación de parámetros hidrológicos en cuencas urbanas. Instituto de Pesquisas Hidráulicas. Universidad Federal de Rio Grande do Sul. Porto Alegre.

- 2008. Estudio de Impacto Ambiental Desagües pluviales en la cuenca alta del $A^{\circ}$ Soto $-3^{\circ}$ Etapa-Nov 2008. Dirección General de Saneamiento y Obras Hidraulicas. Gobierno de la Provincia de Buenos Aires. http://www.ec.gba.gov.ar/UCO/docs/informesambientales/FinanciamientoAdicionalbirf7 268AR/obrasSaneamiento/pluvialesSoto.pdf. Consultado el 25-08-2011

- Angellacio, C., et Al. 2002. Evaluación Preliminar de Impactos Ambientales. Presidencia de la Nación. Secretaría de Obras Públicas. Subsecretaría de Recursos Hídricos. Informe Preliminar.

- Conesa Fernández, V. 1996. Guía metodológica para la Evaluación del Impacto Ambiental. Ed. Mundi Prensa. Madrid.

- INDEC. 2001. Censo Poblacional 2001. Instituto Nacional de Estadísticas y Censos, INDEC. Bs. As.

- Popolizio, E. et Al. 1997. Geociencias. Revista Nordeste. 2da. Época. Serie Investigación y Ensayos. Facultad de Humanidades. UNNE. Bases Conceptuales y Metodológicas. Resistencia, Chaco, Argentina.

- Popolizio, E. 1972. Geomorfología del relieve de Plataforma de la Provincia de Misiones y zonas aledañas. Capitulo I a IV. Anales de la Sociedad Argentina de Estudios Geográficos GÆA, Tomo XV, Buenos Aires.

- Popolizio, E. 1977. Contribución a la Geomorfología de la Provincia de Corrientes. En Geociencias VII y VIII. Publicaciones del Centro de Geociencias Aplicadas. Facultad Ingeniería y Humanidades. UNNE. Resistencia - Chaco - Argentina.

- Popolizio, E. 1982. La geomorfología en los estudios ecológicos de la llanura en Geociencias XII. Publicación del Centro de Geociencias Aplicadas. Universidad Nacional del Nordeste. Resistencia, Chaco, Argentina.

- Popolizio, E. 1983. Teoría General de Sistemas aplicada a la Geomorfología. en Geociencias XI. Publicación del Centro de Geociencias Aplicadas. Universidad Nacional del Nordeste. Resistencia, Chaco, Argentina.

- Popolizio, E. 1987. El enfoque sistémico en la enseñanza de la Geografía. Boletín de la Sociedad Argentina de Estudios Geográficos GÆA No106 Buenos Aires.

- Popolizio, E. 1989. Algunos elementos geomorfológicos condicionantes de la organización espacial y las actividades del NEA. En Geociencias XVII. Publicaciones del Centro de Geociencias Aplicadas. Facultad Ingeniería y Humanidades. UNNE. Resistencia - Chaco - Argentina.

- Foschiatti, A. M. 2004. Vulnerabilidad global y pobreza. Consideraciones conceptuales. En Geografica Digital Año 1- № 2 Julio - Diciembre 2004.

- Foschiatti, A. M. 2006. Vulnerabilidad sociodemográfica del Chaco. EUDENE. Resistencia (Chaco).

Fuentes consultadas

- Subsecretaría de Medio Ambiente. Municipalidad de Corrientes

- Subsecretaría de Planeamiento Urbano. Municipalidad de Corrientes

- Subsecretaría de Obras Públicas. Municipalidad de Corrientes

- Dirección General de Redes Urbanas. Municipalidad de Corrientes

- Dirección General de Higiene Urbana. Municipalidad de Corrientes

Publicado en formato digital: Ing. Guillermo Antonio Arce y Prof. Mgter. Jorge Alfredo Alberto. LA ACTIVIDAD HUMANA Y SU IMPACTO SOBRE LOS SISTEMAS DE DRENAJE URBANOS. ESTUDIO DE CASO: LOS DESAGÜES PLUVIALES DE LA CIUDAD DE CORRIENTES. Revista Geográfica Digital. IGUNNE. Facultad de Humanidades. UNNE. Año 8. № 16. Julio - Diciembre 2011. ISSN 16685180 Resistencia, Chaco. En: http://hum.unne.edu.ar/revistas/geoweb/default.htm 